\title{
EMPLEO DE ZEOLITAS NATURALES EN LA FERTILIZACIÓN Y PRODUCCIÓN DEL FRÉJOL (Phaseolus vulgaris L.) EN LA ZONA DE QUEVEDO
}

\author{
Gorki Teófilo Díaz Coronel ${ }^{1,2}$, Fernando David Sánchez Mora ${ }^{3}$, Luis Tarquino Llerena Ramos y \\ Gregorio Humberto Vásconez Montúfar ${ }^{1}$ \\ ${ }^{1}$ Unidad de Investigación Científica y Tecnológica, Universidad Técnica Estatal de Quevedo, $\mathrm{km} 1$ 1/2 \\ vía Quevedo - Santo Domingo de los Tsáchilas, C. P. 73. Quevedo, Los Ríos, Ecuador. \\ ${ }^{2}$ Facultad de Ciencias Pecuarias, Universidad Técnica Estatal de Quevedo, $\mathrm{km} 7$ vía a El Empalme \\ Mocache, Los Ríos, Ecuador \\ ${ }^{3}$ Facultad de Ciencias Agrarias, Universidad Técnica Estatal de Quevedo, km 1 1/2 vía a Santo Domingo \\ de los Tsáchilas, C. P. 73. Quevedo, Los Ríos, Ecuador.
}

RESUMEN

$\mathrm{C}$ Con el objeto de incrementar la producción y rentabilidad del cultivo de fréjol (Phaseolus vulgaris L.), se evaluaron dos tipos de zeolitas comerciales: "Zeolite C" y "Roca Mágica", ambas del tipo Clinoptilolita, en dosis de 25,50 y $75 \%$ de la fertilización recomendada para el cultivo en la localidad de Quevedo (80-40-00). Se utilizó la variedad de fréjol "EVG-6". Los tratamientos se generaron de forma factorial, 2 (tipos de zeolita) $\mathrm{x}$ 3 (dosis de zeolita) +1 (testigo fertilización sin zeolita), y se los dispuso de un diseño de bloques completos al azar con cuatro repeticiones. Para comparar los tratamientos se utilizó la prueba de Tukey $(\mathrm{P} \leq 0.05)$. En la parcela útil se midió: días a floración, daño de plagas y enfermedades, número de vainas por planta, número de granos por vaina, peso de 100 semillas, rendimiento por planta y por hectárea. Con la mayor dosis de zeolita se adelantó la floración del fréjol aproximadamente dos días. El menor daño de las plagas y enfermedades se registró con la aplicación de la zeolita natural en conjunto con los fertilizantes. Entre los tipos de zeolita solo se encontraron diferencias estadísticas en el peso de 100 semillas y rendimiento por planta; en las otras variables no se presentaron diferencias estadísticas. En el contraste: "zeolita vs sin zeolita" se encontró una mayor producción por planta y por hectárea a favor de la aplicación de la zeolita. Con el empleo del fertilizante más " $75 \%$ de Zeolite - C" y " $25 \%$ de Roca Mágica" se obtuvieron las mayores rentabilidades.

Palabras claves: zeolita, fréjol, Phaseolus vulgaris L., fertilizante, suelo.

\section{Abstract}

$\mathrm{I}^{\mathrm{n}}$ order to increase production and profitability of common bean (Phaseolus vulgaris L.) crop, two types of commercial zeolites were assessed: "Zeolite C" and "Roca Magica" both of the Clinoptilolite type, in doses of 25,50 and $75 \%$ of the recommended fertilization (8040-00) for this crop in Quevedo city area. The variety of common bean used was "EVG-6". The treatments were generated in the factorial form as follows 2 (types of zeolite) x 3 (doses of zeolite) +1 (fertilization without zeolite as control), arranged in a Complete Randomized Blocks Design with four replications. To compare the means of the treatments, the Tukey test was used $(\mathrm{P} \leq$ 0.05 ). Days to flowering, pests and diseases damages number of pods per plant, number of grains per pod, weight of 100 seeds and yield per hectare was measured in the plot. With the highest dose of zeolite along with chemical fertilizers an early flowering of about two days was observed. The minor pests and diseases damage was registered with the application of natural zeolite along with fertilizers. Among the types of zeolite statistical differences were found only in weight of 100 seeds and yield per plant; no statistical differences were found in the other variables. In the "zeolite vs. without zeolite" contrast a major production per plant and per hectare was found in favor of the application of zeolite. The higher profitability was obtained with the use of fertilizer plus " $75 \%$ of Zeolite C" and " $25 \%$ of Roca Magica".

Key words: zeolite, bean, Phaseolus vulgaris L., fertilizer, soil.

\section{INTRODUCCIÓN}

$\mathrm{E}_{\mathrm{d}}^{\mathrm{l}}$ proceso de degradación de la tierra, es concebido de manera natural o inducido por el hombre, es la disminución o destrucción del potencial biológico de los recursos naturales ocasionada por el mal uso y manejo de los mismos. Algunos de los factores que favorecen este proceso son la expansión demográfica, la sobre explotación de los recursos naturales, el indiscriminado uso de sales fertilizantes entre otras no menos importantes (Guevara, s.f.).

Por su parte, las zeolitas mejoran las propiedades químicas y físicas del suelo, es uno de los caminos más efectivos para incrementar la capacidad de

Recibido: Enero, 2009. Aceptado: Febrero, 2009.

Publicado como ARTÍCULO en Ciencia y Tecnología 3: 1-6. 2009. intercambio catiónico (CIC) en la zona de las raíces y disminuir las aplicaciones de fertilizantes reduciendo las pérdidas por volatilización y lixiviación de los de los mismos (Zivadinovic, 1994., citado por Coello y Osorio, 2003). Además, contribuye ostensiblemente a mejorar la humedad de la capa arable, facilita la fluidez del agua y reduce la densidad aparente del suelo, lográndose un incremento de producción en la cosecha de los cultivos, y sobre todo, reduce el impacto ecológico. La zeolita no actúa como fertilizante, sino como un aditivo que permite incrementar la eficiencia de los fertilizantes, permitiendo una disponibilidad controlada de los cationes que son utilizados por las plantas en su nutrición (Iskenderov, s.f., citado por Montalvo et al., 
1991).

En el caso del fréjol (Phaseolus vulgaris L.), se ha reportado incrementos en los rendimientos en el orden del $10 \%$, cuando se adiciona zeolita de forma conjunta con el fertilizante (fertilizante $+25 \%$ de zeolita) (CEPIS, s.f.), lo cual se atribuye a la alta CIC que poseen las zeolitas, gran poder de adsorción y capacidad de hidratación y deshidratación sin afectar su estructura y propiedades que incrementan la eficiencia de los fertilizantes.

En este contexto, esta investigación fue desarrollada en Quevedo con el aval de la Unidad de Investigación Científica y Tecnológica (UICYT), teniendo como objetivo establecer el tipo y la dosis de zeolita que permita incrementar el rendimiento en la variedad de fréjol "EVG-6".

\section{Materiales y Métodos}

$\mathrm{L}$ a presente investigación se realizó en la época seca del año 2006, durante los meses de agosto a octubre, en la finca experimental "La María" de la Universidad Técnica Estatal de Quevedo, ubicada en el km 7, Vía Quevedo - El Empalme, cuyas coordenadas geográficas son: $79^{\circ} 27^{\prime}$ de longitud Oeste y $01^{\circ} 06^{\prime}$ de latitud Sur, a una altitud de $73 \mathrm{msnm}$. La fase del cultivo tuvo una duración de 80 días.

\section{Analisis de suelo}

El ensayo se estableció en un Andisol. Para una adecuada fertilización se realizó un análisis del suelo donde se estableció el experimento. Se encontró que el suelo era rico en potasio pero deficiente en nitrógeno y fósforo, con un alto contenido de materia orgánica y textura franco arcillosa, clasificado como un suelo apto para la agricultura.

\section{Material genético y fertilizantes empleados}

El material genético utilizado fue la variedad de fréjol: "EVG-6" de Ecuavegetal y como fuente de nitrógeno y fósforo se utilizó urea $(46 \% \mathrm{~N})$ y súper fosfato triple $\left(46 \% \mathrm{P}_{2} \mathrm{O}_{5}\right)$ a los que se le adicionó dos tipos de zeolitas comerciales: "Zeolite C" y "Roca Mágica", en tres dosis: 25,50 y $75 \%$ del peso de la fertilización convencional.

\section{Diseño experimental y análisis estadístico}

Se utilizó un diseño de bloques completos al azar en arreglo factorial: dos tipos de zeolitas ("Zeolite C" y "Roca Mágica") por tres dosis (25, 50 y $75 \%)$, más un testigo (dosis de fertilizante químico sin zeolita, de acuerdo al análisis de suelo) con cuatro repeticiones. Para las comparaciones entre medias de tipos y dosis de zeolita se empleó la prueba de Tukey al 5\% de probabilidad. Además, se realizaron contrastes ortogonales entre grupos de tratamientos. El modelo lineal fue el siguiente: $y_{i j k}=\mu+\tau_{i}+\alpha_{j}+\beta_{k}+(\alpha \beta)_{j k}+e_{i j k}$

Donde: $y_{i j k}=$ Valor de la característica en estudio debido al bloque $i$, al tipo de zeolita $j$, y a la dosis de zeolita $k ; \mu=$ Efecto común de todas las observaciones; $\tau_{i}=$ Efecto del bloque $i ; \alpha_{j}=$ Efecto del tipo de zeolita $j$; $\beta_{k}=$ Efecto de la dosis de zeolita $k ;(\alpha \beta)_{i j}=$ Efecto de la interacción entre tipo $j$ y dosis de zeolita $k$; $e_{i j k}=$ Error de observación sobre la unidad experimental $i j k$.

\section{Tratamientos y especificaciones de siembra}

Los siete tratamientos evaluados, resultaron de la combinación de los factores tipos de zeolita ("Zeolite-C" y "Roca Mágica") y dosis de zeolita: (25, 50, y $75 \%$ ), más un testigo (Fertilización sin zeolita) (Cuadro 1).

Se conformaron unidades experimentales con cuatro surcos de $6.0 \mathrm{~m}$ de largo, con $0.5 \mathrm{~m}$ de separación, con una distancia entre planta de $0.2 \mathrm{~m}$, y la separación entre unidades experimentales de un mismo bloque y entre bloque fue de 1.0 y $2.0 \mathrm{~m}$, respectivamente. Como parcela útil se utilizaron los dos surcos centrales

Cuadro 1. Tratamientos, producto de la combinación de los factores tipo y dosis de zeolita

\begin{tabular}{ccc}
\hline \multirow{2}{*}{$\mathbf{N}^{\mathbf{0}}$} & \multicolumn{2}{c}{ Tratamientos } \\
\cline { 2 - 3 } & Tipos de zeolita & Dosis (\%) \\
\hline 1 & Zeolita $-\mathrm{C}$ & 25 \\
2 & Zeolita $-\mathrm{C}$ & 50 \\
3 & Zeolita $-\mathrm{C}$ & 75 \\
4 & Roca Mágica & 25 \\
5 & Roca Mágica & 50 \\
6 & Roca Mágica & 75 \\
7 & Sin zeolita & - \\
\hline
\end{tabular}

\section{Fertilización}

La fertilización se la hizo con base en N y P, con la finalidad de tener una buena disposición de los nutrientes para las plantas. Se fraccionó la aplicación en dos partes: la primera fertilización se la hizo a los ocho días después de la siembra con $40 \mathrm{~kg} \mathrm{ha}^{-1}$ de $\mathrm{N}$ (urea) y $40 \mathrm{~kg} \mathrm{ha}^{-1}$ de $\mathrm{P}$ (súper fosfato triple), que corresponde al $50 \%$ del nitrógeno y el $100 \%$ del fósforo, más la zeolitas en dosis del 25,50 y $75 \%$ del peso de los fertilizantes. La segunda fertilización se la hizo a los 25 días después de la siembra, donde aplicando los $40 \mathrm{~kg} \mathrm{ha}^{-1}$ de $\mathrm{N}$ res- 
tante.

\section{Mediciones experimentales}

Se tomaron datos de las variables: días a floración, altura de planta, incidencia de plagas y enfermedades, vainas por planta, granos por vaina y por planta, tamaño de vaina, peso de 100 semillas, rendimiento por planta y por hectárea. Además, se analizó la rentabilidad de los tratamientos.

\section{Resultados y Discusión}

$\mathrm{E}^{\mathrm{n}}$ la etapa de floración no se reportaron diferencias estadísticas para el tipo de zeolita $(\mathrm{P}>0.05)$. Sin embargo, para dosis de zeolita sí se presentaron diferencias entre la dosis del 25 y 75\%. Hubo un efecto lineal negativo significativo $(b=-0.03768)$, indicando que a medida que se incrementó la dosis de zeolita disminuyó los días a floración. Se observó que con el 75\% de zeolita se adelanta la floración en aproximadamente dos días (Cuadro 2, Figura 1). Asimismo, el contraste ortogonal en el cultivo de fréjol, "zeolita vs sin zeolita", se determinó diferencias entre grupos, observándose que la aplicación de los fertilizantes conjuntamente con la zeolita disminuye los días a floración en relación con la no aplicación de zeolita (Cuadro 3).

Lo anterior indica que la adición de zeolita conjuntamente con el fertilizante edáfico, acelera los días a floración en el fréjol, lo cual posiblemente se deba a que la zeolita mejora las propiedades químicas y físicas de los suelos (Iskenderov, s.f., citado por Montalvo et al., 1991), favoreciendo la nutrición de las plantas, y por ende, un normal metabolismo de las mismas (Padilla, 2005).

Cuadro 2. Días a floración de la variedad de fréjol "EVG - 6", bajo el efecto de dos tipos y tres dosis de zeolita

\begin{tabular}{lrl}
\hline \multicolumn{1}{c}{ Tipos y dosis } & Floración $^{\dagger}$ & \\
\hline Tipos de zeolita & & \\
Zeolite - C & 28.33 & a \\
Roca Mágica & 28.42 & a \\
Dosis de zeolita & & \\
$25 \%$ & 29.38 & a \\
$50 \%$ & 28.25 & ab \\
$75 \%$ & 27.50 & $\mathrm{~b}$ \\
\hline $\bar{X}$ & 28.38 & \\
CV (\%) & 2.51 & \\
\hline
\end{tabular}

Promedios con letras diferentes presentan diferencias estadísticas, de acuerdo a la prueba de Tukey $(\mathrm{P} \leq 0.05)$.

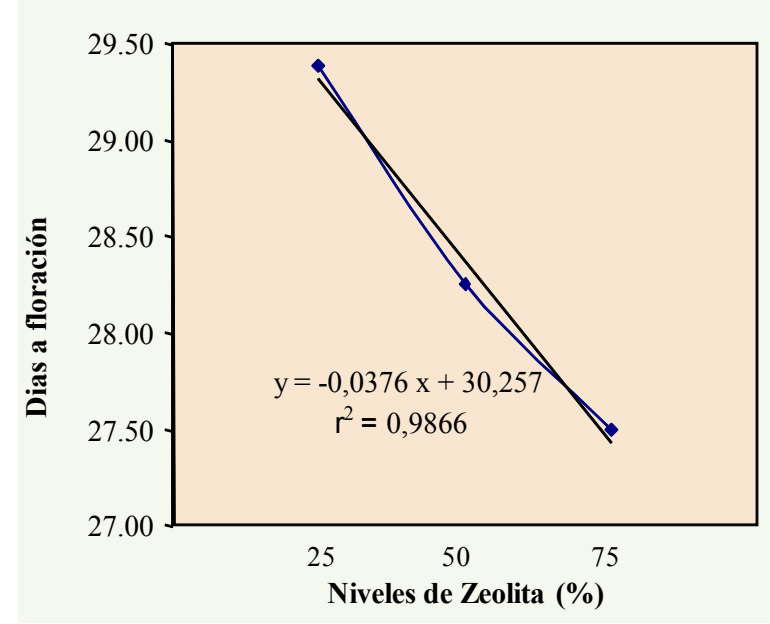

Figura 1. Regresión lineal simple, entre los días a floración de la variedad de fréjol "EVG-6" (y), bajo el efecto de tres dosis de zeolita (x).

Cuadro 3. Contraste entre tratamientos con zeolita vs sin zeolita, en los días a floración de la variedad de fréjol "EVG-6", bajo el efecto de dos tipos y tres dosis de zeolita

\begin{tabular}{cc}
\hline Contraste & Floración $^{\dagger}$ \\
\hline Con zeolita & $28.38 \mathrm{~b}$ \\
vs &
\end{tabular}

Sin zeolita ( $\mathrm{t}$ )

30.25 a

$\dagger$ Promedios con letras diferentes presentan diferencias estadísticas, de acuerdo a la prueba de $\mathrm{F}(\mathrm{P} \leq 0.05)$.

$\mathrm{t}=$ testigo

El daño foliar por insectos y enfermedades, fue valorado de acuerdo a la escala de daño de 1-5, propuesta por Quijije (2006), siendo: 1, ninguno; 2, leve; 3, moderado; 4, severo y 5, muy severo. El contraste ortogonal en el cultivo de fréjol, "zeolita vs sin zeolita", para el daño de insectos plagas, no presentó diferencias estadísticas $(\mathrm{P}>0.05)$; en cambio, los promedios de daño por enfermedades fueron estadísticamente diferentes $(\mathrm{P} \leq 0.05)$. El daño foliar por plagas fue de 2.58 y 3.00, $\mathrm{y}$ para el caso de daño foliar por enfermedades fue de 2.41 y 3.50, respectivamente (Cuadro 4). El menor daño por enfermedades se registró con la utilización de zeolita en relación a los tratamientos fertilizados de forma normal, lo que concuerda con Páez (2006), quien indica, que los cultivos que se desarrollan en suelos donde se han hecho aplicaciones de zeolita, evidencian un mejor estado nutricional, disminuyendo el ataque de plagas y enfermedades. 
Cuadro 4. Contraste entre tratamientos con zeolita vs sin zeolita, en el daño foliar por insectos plagas y enfermedades en la variedad de fréjol "EVG - 6", bajo el efecto de dos tipos y tres dosis de zeolita

\begin{tabular}{|c|c|c|}
\hline Contraste & Insectos plagas $^{\dagger}$ & Enfermedades $^{\dagger}$ \\
\hline Con zeolita & $2.58 \mathrm{a}$ & $2.42 \mathrm{~b}$ \\
\hline \multicolumn{3}{|l|}{ vs } \\
\hline Sin zeolita (t) & $3.00 \mathrm{a}$ & $3.50 \mathrm{a}$ \\
\hline
\end{tabular}

Al evaluar el efecto del tipo de zeolita, se reportaron diferencias estadísticas para el peso de $100 \mathrm{se}-$ millas y rendimiento por planta, donde la "Zeolite - C" reportó los mayores valores con 43.54 y 17.16 g, respectivamente. Además, al evaluar el efecto de la dosis de zeolita, se determinó que el 25\% de zeolita permite alcanzar los mayores valores para número de granos por vaina, peso de 100 semillas, rendimiento por planta y por hectárea, a pesar de no ser estadísticamente diferente con respecto a las dosis de 50 y $75 \%$ (Cuadro 5).

Cuadro 5. Número de vainas por planta, granos por vainas, peso de 100 semillas, rendimiento por planta y por hectárea de la variedad de fréjol "EVG - 6", bajo el efecto de dos tipos y tres dosis de zeolita

\begin{tabular}{cccccc}
\hline Tipos y dosis & $\begin{array}{c}\text { Número de } \\
\text { vainas por } \\
\text { planta }\end{array}$ & $\begin{array}{c}\text { Número de } \\
\text { granos por } \\
\text { vaina }\end{array}$ & $\begin{array}{c}\text { Peso de 100 } \\
\text { semillas } \\
(\mathbf{g})\end{array}$ & $\begin{array}{c}\text { Rendimiento por } \\
\text { planta } \\
(\mathbf{g})\end{array}$ & $\begin{array}{c}\text { Rendimiento por } \\
\text { hectárea } \\
(\mathbf{k g})\end{array}$ \\
\hline Tipos de zeolita & & & & & \\
Zeolite-C & $11.42 \mathrm{a}^{\dagger}$ & $3.52 \mathrm{a}$ & $43.54 \mathrm{a}$ & $17.16 \mathrm{a}$ & $1330.21 \mathrm{a}$ \\
$\quad \begin{array}{l}\text { Roca Mágica } \\
\text { Dosis de zeolita }\end{array}$ & $10.52 \mathrm{a}$ & $3.53 \mathrm{a}$ & $42.13 \mathrm{~b}$ & $15.36 \mathrm{~b}$ & $1255.87 \mathrm{a}$ \\
& & & & & \\
$25 \%$ & $11.35 \mathrm{a}$ & $3.67 \mathrm{a}$ & $42.64 \mathrm{a}$ & $16.60 \mathrm{a}$ & $1374.15 \mathrm{a}$ \\
$50 \%$ & $10.56 \mathrm{a}$ & $3.33 \mathrm{a}$ & $43.23 \mathrm{a}$ & $16.04 \mathrm{a}$ & $1195.76 \mathrm{a}$ \\
$75 \%$ & $10.99 \mathrm{a}$ & $3.58 \mathrm{a}$ & $42.63 \mathrm{a}$ & $16.14 \mathrm{a}$ & $1309.18 \mathrm{a}$ \\
\hline $\bar{X}$ & 10.97 & 3.53 & 42.83 & 16.26 & 1293.03 \\
$\mathbf{C V}(\mathbf{\%})$ & 15.27 & 6.54 & 3.87 & 12.31 & 21.03 \\
\hline
\end{tabular}

${ }^{\dagger}$ Promedios con letras diferentes presentan diferencias estadísticas, de acuerdo a la prueba de Tukey $(\mathrm{P} \leq 0.05)$.

En el contraste ortogonal "zeolita vs sin zeolita", el peso de 100 semillas de la variedad de fréjol "EVG - 6" no mostró diferencias estadísticas entre grupos de tratamientos; en cambio, en el rendimiento por planta y por hectárea fue mayor en las parcelas tratadas con zeolita a cuando no se aplicó zeolita conjuntamente con los fertilizantes $(\mathrm{P} \leq 0.05)$ (Cuadro 6). Se confirma así, que la zeolita incrementa el nivel de rendimiento de las plantas, ya que mejora la eficiencia de los fertilizantes (Coello y Osorio, 2003). La zeolita aumenta la disposición de nutrientes, incrementado así, la productividad del fréjol (Martínez, 2005). 
Cuadro 6. Contraste entre tratamientos con zeolita vs sin zeolita, en el peso de 100 semillas, rendimiento por planta y por hectárea de la variedad de fréjol "EVG - 6", bajo el efecto de dos tipos y tres dosis de zeolita

\begin{tabular}{|c|c|c|c|}
\hline Contraste & $\begin{array}{c}\text { Peso de } 100 \\
\text { semillas }^{\dagger} \\
\text { (g) } \\
\end{array}$ & $\begin{array}{c}\text { Rendimiento } \\
\text { por planta }^{\dagger} \\
\text { (g) }\end{array}$ & $\begin{array}{c}\text { Rendimiento } \\
\text { por hectárea } \\
(\mathrm{kg})\end{array}$ \\
\hline $\begin{array}{c}\text { Con zeolita } \\
\text { vs }\end{array}$ & $42.83 \mathrm{a}$ & $16.26 \mathrm{a}$ & $1293.03 \mathrm{a}$ \\
\hline Sin zeolita $(\mathrm{t})$ & $41.72 \mathrm{a}$ & $13.56 \mathrm{~b}$ & $994.00 \mathrm{~b}$ \\
\hline
\end{tabular}

${ }^{\dagger}$ Promedios con letras diferentes presentan diferencias estadísticas, según prueba de $\mathrm{F}(\mathrm{P} \leq 0.05)$ $\mathrm{t}=$ testigo.

En cuanto a la rentabilidad, se observó que los tratamientos con 25\% de "Roca Mágica" y 75\% de "Zeolite - C" reportaron las mayores rentabilidades, los mismos fueron fertilizados con $80 \mathrm{~kg} \mathrm{ha}^{-1}$ de $\mathrm{N}$ y $40 \mathrm{~kg}$ ha $^{-1}$ de P (Figura 2).

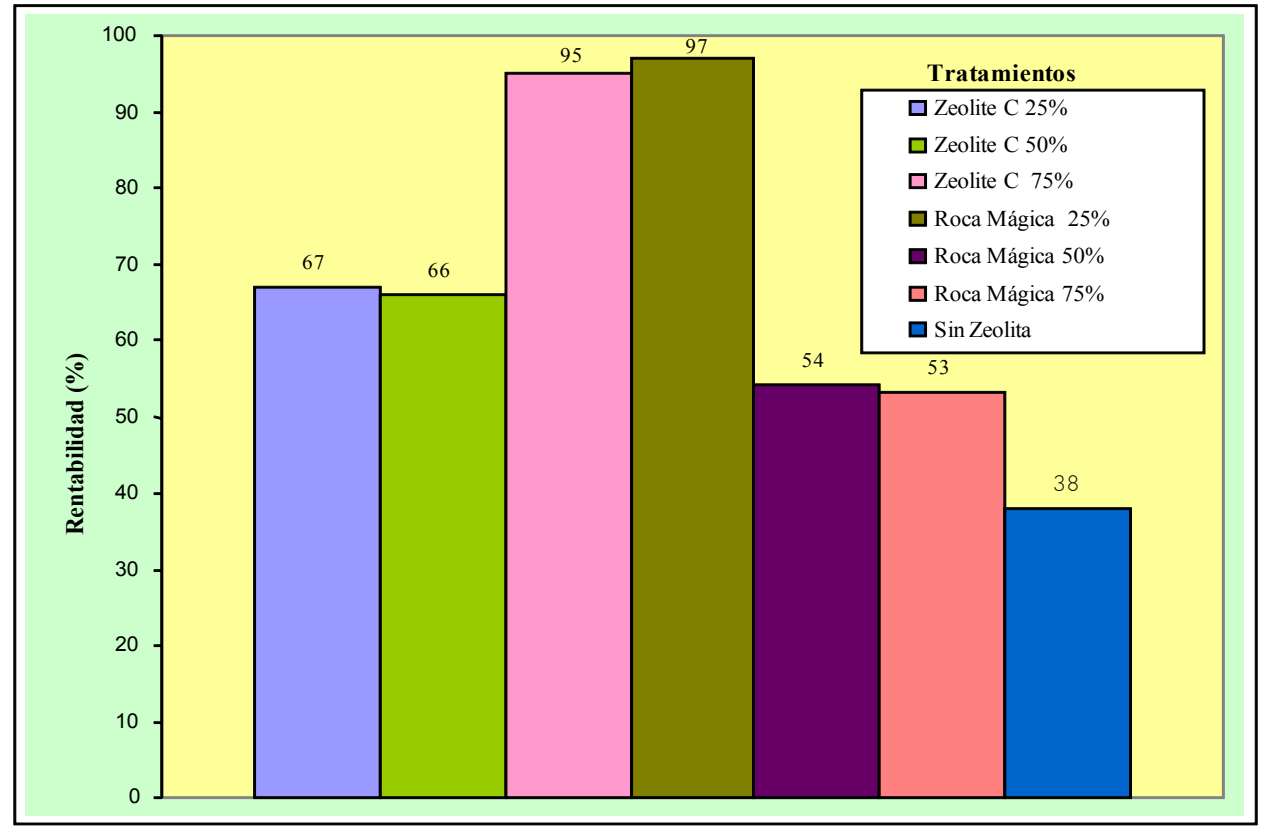

Figura 2. Rentabilidad (\%) de la variedad de fréjol "EVG - 6", bajo el efecto de dos tipos y tres dosis de zeolita

\section{Conclusiones}

$\mathrm{D}^{\mathrm{s}}$ el análisis de los resultados y de la discusión de los mismos se concluyó lo siguiente:

En el cultivo del fréjol, variedad "EVG - 6", la dosis de $75 \%$ de zeolita reduce los días a floración en un $6 \%$ con respecto a la dosis de $25 \%$.

Los daños por plagas y enfermedades, en la variedad de fréjol "EVG-6", es menor cuando se aplica fertilizante edáfico combinado con zeolita.

El mayor peso de 100 semillas y rendimiento por planta de la variedad de fréjol "EVG - 6" se obtuvo con "Zeolite C".

La aplicación de zeolita en conjunto con la fertilización edáfica, origina un incremento en el rendimiento por planta y por hectárea en comparación con el testigo, en el que solo se utilizó fertilización convencional.

De acuerdo con el análisis económico basado en el rendimiento de la variedad de fréjol "EVG - 6", la mayor producción y rentabilidad por hectárea, se alcanzó con el 25\% de "Roca Mágica" y 75\% de "Zeolite - C" en conjunto con la fertilización de $80 \mathrm{~kg} \mathrm{ha}^{-1}$ de $\mathrm{N}$ y $40 \mathrm{~kg} \mathrm{ha}^{-1}$ de $\mathrm{P}$. 


\section{Literatura Citada}

CEPIS (Centro Panamericano de Ingeniería Sanitaria y Ciencias del Ambiente, PE). s.f. Las zeolitas en la agricultura (en línea). Consultado el 13 de noviembre del 2008. Disponible en http://www. cepis.ops-oms.org/bvsacd/scan2/032752/03275202.pdf

Coello, H.; Osorio, L. 2003. Estudio comparativo de fertilización orgánica y química en mezcla con zeolita en el cultivo de pimiento (Capsicum anhum L.) en la zona de Quevedo. Tesis Ing Agr. Quevedo, EC. Facultad de Ciencias Agrarias. Universidad Técnica Estatal de Quevedo. 63 p.

Guevara, R. s.f. Impacto del cambio de uso del suelo en el proceso erosivo. Centro Universitario de la Costa Sur de la Universidad de Guadalajara. Jalisco, MX. 2 p.

Martínez, S. 2005. Evaluación del Transporte de Nutrientes en la Aplicación Agrícola de Biosólidos con Nutrientes y Zeolitas. (En línea). Consultado el 21 de febrero del 2006. Disponible en http:// cbi.azc.uam.mx/coord/cdd/08/proyectoterminal / scripts/getdata2.php? $\mathrm{id}=74$
Montalvo, H.; Diaz, H.; Valenzuela R y Umpierrez C. 1991. Memorias de la 3ra. Conferencia Internacional sobre Ocurrencia, Propiedades y Usos de las Zeolitas Naturales. Habana, CU. p. 74.

Padilla, W. 2005. Fertilización de suelos. Grupo clínica agrícola. 4 ed. Quito, EC. CD - R. Formato PDF. $185 \mathrm{p}$.

Páez, O. 2006. Una necesaria introducción sobre las Zeolitas. (En línea). Consultado el 22 de mayo del 2007. Disponible en: http://www.soil-fertility. com/zeolite/ espagnol/index.shtml

Quijije, R. 2006. Valoración del daño foliar por insectos y enfermedades en el cultivo de fréjol (entrevista). Estación Experimental Tropical Pichilingue, INIAP. Los Ríos, EC. 